\title{
Poled Polyaniline Coated Piezo Composite Using Low Electric Field and Reduced Poling Time: A Functional Material
}

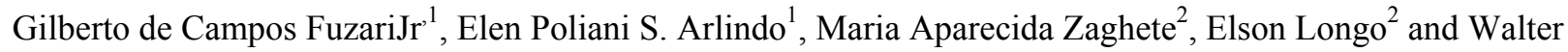 \\ Katsumi Sakamoto ${ }^{3}$ \\ 1. Federal University of Mato Grosso, Campus of Araguaia, Institute of Exact and Earth Sciences, Barra do Garças (MT), Brazil \\ 2. Chemistry Institute of Araraquara, Department of Biochemistry and Chemistry Technology, São Paulo State University - UNESP, \\ Brazil
}

3. School of Enginnering, Department of Physics and Chemistry, São Paulo State University, UNESP, Campus of Ilha Solteira, Av. Brasil 56, IlhaSolteira (SP) 15385-000, Brazil

Received: April 02, 2014 / Accepted: April 14, 2014 / Published: April 25, 2014.

\begin{abstract}
Composite films were prepared by two different routes: lead zirconate titanate (PZT) particles coated with polyaniline (PAni) dispersed in a polymeric matrix of polyvinylidene fluoride (PVDF); and PZT particles and PAni powder dispersed separately in the polymer matrix. The electrical conductivity of the particles was controlled by the protonation and de-protonation of PAni in solution with controlled $\mathrm{pH}$. The results indicate that the percolation threshold of the composite made of PZT coated with a conductive layer (PZT-PAni) is in the range of 20 vol.\% to 30 vol.\% of PZT-PAni. The PZT-PAni/PVDF composite redoped in solution with pH 3.7 showed the best results in terms of longitudinal piezoelectric coefficient $\left(d_{33}\right)$ in samples containing 30 vol.\% of ceramic particles due to the equilibrium between conduction and poling effects on the composite. The poling process of the composite sample required just $5 \mathrm{MV} / \mathrm{m}$ electric field applied during $15 \mathrm{~min}$. Furthermore, the composite was used as sensor in structural health monitoring (SHM), showing the possibility to propose it as a functional material.
\end{abstract}

Key words: Piezo-composite, controlled conductivity, polarization, PZT coated particles, electrical flux path.

\section{Introduction}

Ferroelectric ceramic polymer composites dispersed in polymer matrices are a well-established alternative to lithium-based crystals and ferroelectric polymers. The main advantage of composite materials over individual ones is that they enable the ceramic's high piezoelectric and pyroelectric activities to be combined with the polymer's excellent mechanical properties at the desired ratios and for specific applications [1,2].

Numerous papers have described the preparation and characterization of ceramic/polymer composites, most

Corresponding author: Walter Katsumi Sakamoto, Dr., professor, research fields: piezoelectric sensors, energy harvesting. E-mail: sakamoto@dfq.feis.unesp.br. of them aimed at their application as sensors, transducers or actuators [3-6]. The findings published in the literature indicate that ceramic/polymer composites have potential technological applications in several areas because they can be obtained as flexible film. However, despite the advantages of composite materials, their poling still poses difficulties $[1,7]$ because the effective electric field to polarize ferroelectric ceramic particles is much lower than the applied electric field.

The poling efficiency of ceramic particles dispersed in a polymer matrix can be enhanced by introducing a third phase into the composite to create an electrical flux path between the ferroelectric particles. Several 
studies have shown that the inclusion of a semiconductor phase favors the poling process [6-12]. However, the effect of a semiconductor phase on the polarization state and ferroelectric property of the composite is still not well-known and requires more in-depth studies.

In this study, a conducting polymer (polyaniline, PAni) was included in the composite to control its electrical conductivity, using two distinct routes: (a) partial coating of the ceramic particles, and (b) dispersion of the PAni powder separately from the PZT powder. The longitudinal piezoelectric coefficient, $d_{33}$, was measured to compare the electroactive properties of the composite films obtained by the two above described routes. The aim was to understand the advantages of fabricating composites using particles partially coated with a conductive layer, and to determine how the poling process occurs in this case.

\section{Experiments}

\subsection{Samples}

Composite films were prepared by two distinct routes. In the first route, PZT, PAni and PVDF ( $\alpha$ form) powders were mixed together mechanically and pressed in the melting temperature range of PVDF, resulting in the $\mathrm{PZT} / \mathrm{PAni} / \mathrm{PVDF}$ composite. In the second route, PZT particles were partially coated with PAni and then mixed with PVDF and pressed as described by Fuzari Jr. [8], resulting in PZT-PAni/PVDF composite film.

\subsection{Measurements}

(a) DC conductivity was measured at room temperature, using a Keithley 236 Source Measure Unit (SMU);

(b) FEG-SEM micrographs were recorded, using a JEOL model 7500F scanning electron microscope;

(c) Electrical Impedance was measured in disc-shape PZT-PAni samples prepared from PZT particles partially coated with PAni. The composite film was not subjected to this analysis due to the low dielectric constant exhibited by the PVDF matrix, which could mask the occurrence of the pseudo-connection of the conductive phase;

(d) Piezoelectric Coefficient was measured in composite samples previously polarized with an electric field of $5.0 \mathrm{MV} / \mathrm{m}$ for $15 \mathrm{~min}$ at $90^{\circ} \mathrm{C}$. The piezoelectric activity was analyzed by measuring the longitudinal piezoelectric coefficient $d_{33}$, using a Trek 610C power supply, a Pennebaker Model 8000 Piezo d33 Tester (American Piezo Ceramics, Inc.) and a HP 34401A multimeter;

(e) Acoustic Emission (AE) tests were carried out with the composite film surface mounted onto an aluminum plate $(300 \times 300 \times 1 \mathrm{~mm})$. To simulate the AE source, pencil lead was broken on the surface of the plate. This is the conventional Hsu-Nielsen [13] method which obeys the ASTM standards.

\section{Results and Discussion}

PZT-PAni/PVDF composite films were produced with different thicknesses according to the ceramic-to-polymer ratio. The higher the ceramic content the greater the thickness and the more brittle the sample. Film thicknesses ranged from $100 \mu \mathrm{m}$ to $500 \mu \mathrm{m}$. Samples with low ceramic content are thinner and more flexible. Also, they are more likely to possess 0-3 connectivity [14], i.e., the ceramic particles are dispersed separately in the polymer matrix and are not connected to each other. On the other hand, the electroactive properties of these samples are reduced due to their lower dipole density.

PZT-PAni containing 30 vol.\% of ceramic showed a uniform distribution in the matrix, with connectivity close to 0-3, as illustrated in Fig. 1a. The presence of PAni-coated PZT particles can be observed in Fig. 1b, which shows a thin layer of conducting polymer on the particles. At 50 vol.\% of ceramic, the particles are highly interconnected, as indicated in Fig. 2. In this case, the composite film can no longer be considered to possess 0-3 connectivity. 


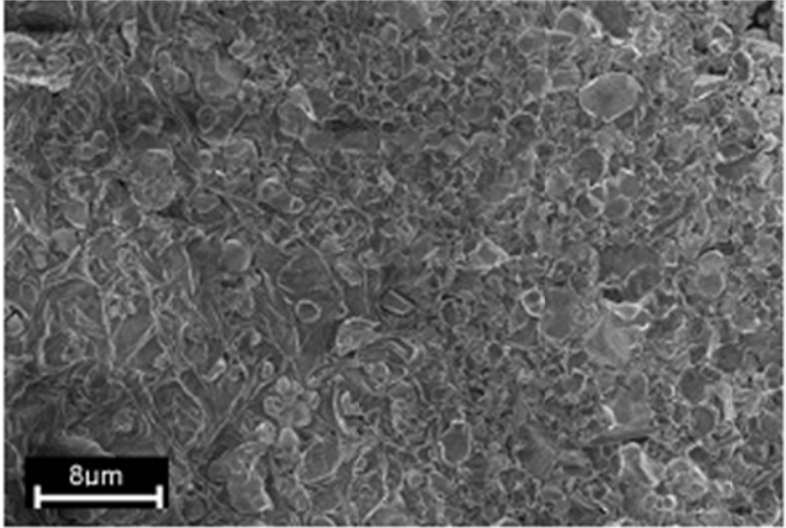

(a)

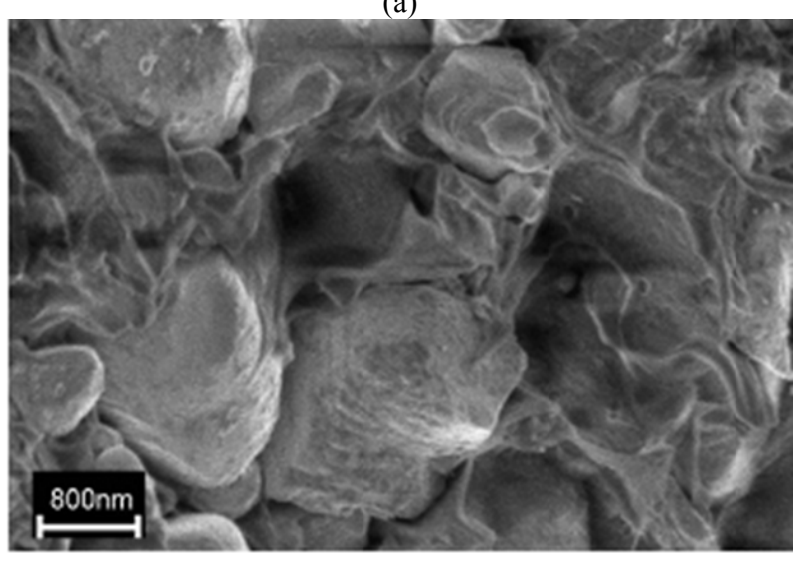

(b)

Fig. 1 FEG images (profile) of PZT-PAni/PVDF 30/70 vol.\%: (a) distribution of particles in the matrix, (b) thin layer of PAni coating on PZT particles.

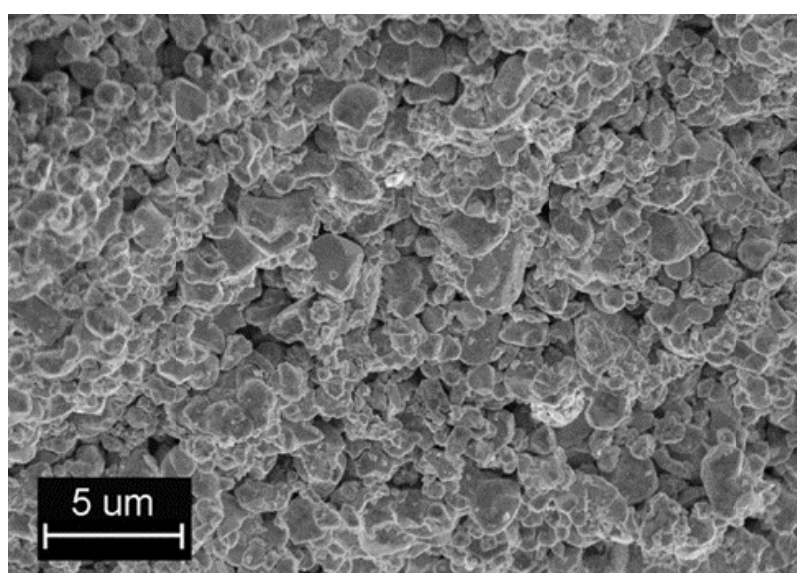

Fig. 2 Distribution of ceramic particles in the PZT-PZni/PVDF 50/50 vol.\% composite.

Fig. 3 shows the behavior of the electrical conductivity of the PZT-PAni/PVDF composite. The percolation threshold (critical concentration) [15] is reached at around 30 vol.\%. Composite samples with higher PZT- PAni content exhibit mixed connectivity ranging from $0-3$ to $3-3$.

In contrast, when conducting polymer (PAni) powder is dispersed in PVDF matrix as a third phase (separately from PZT particles), the percolation threshold occurs between 0.5 and 1.0 vol.\% of PAni, as shown in Fig. 4. Note that the electrical conductivity of the particles in the PZT-PAni composite was one order of magnitude lower than in the sample containing PAni powder dispersed as a third phase in the polymer matrix. This can be attributed to the physical interaction of aniline with PZT, which can limit chain size during the polymerization of PAni. Short chainsdisplay reduced intrachain conduction (polaron, bipolaron model) and enhanced interchain conduction (hopping or tunneling) [16, 17]. Furthermore, because

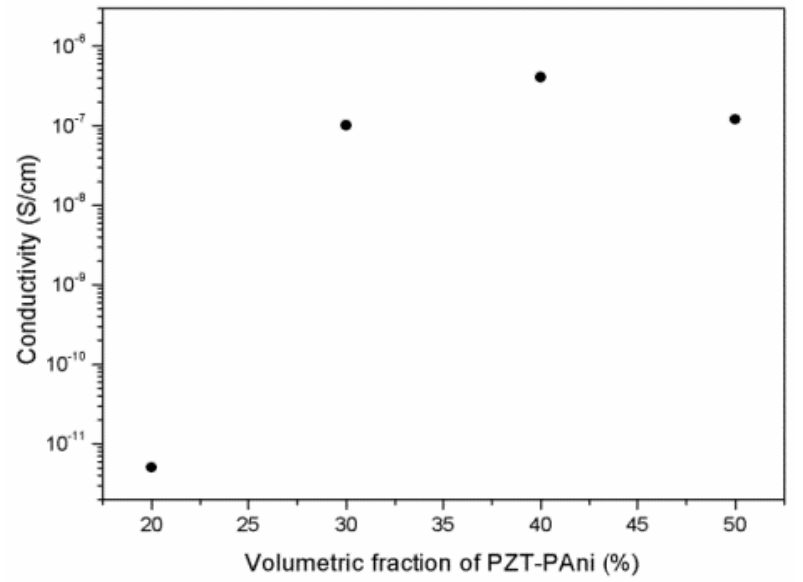

Fig. 3 Electrical conductivity of PZT-PAni/PVDF composite as a function of ceramic content.

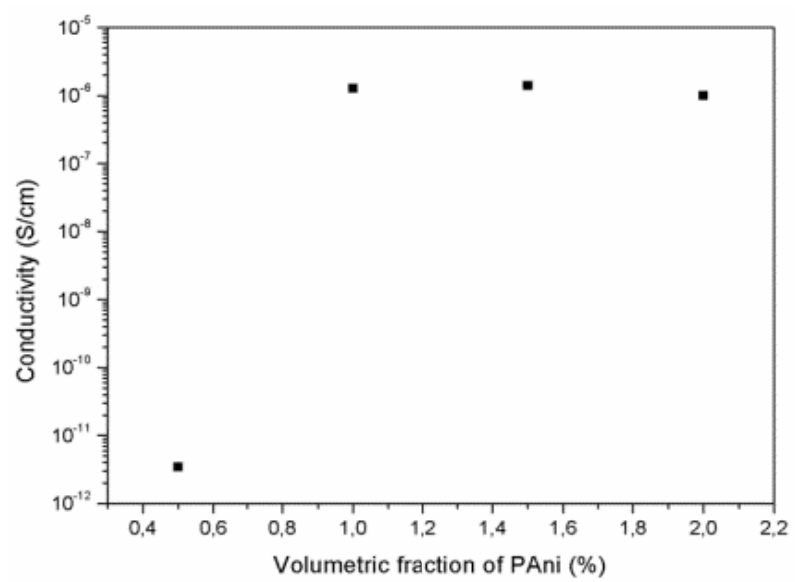

Fig. 4 Electrical conductivity of PZT/PAni/PVDF composite. The PAni was dispersed in polymer matrix separately from the PZT. The volume fraction of PVDF was $70 \%$. 
the PZT particles are not completely coated, the PAni molecules may be separated by a small distance, resulting in the appearance (or increase) of small potential barriers, even above the percolation threshold. In other words, although the ceramic particles appear to be connected (pseudo-connection), they are separated by small distances from the conducting material adhering to them [17].

The PZT/PAni/PVDF composite containing 29.5/0.5/70 vol.\% was poled at $90{ }^{\circ} \mathrm{C}$ for $15 \mathrm{~min}$ with 5.0 MV/m dc electric field and the measured longitudinal piezoelectric coefficient $d_{33}$ was $7.0 \mathrm{pC} / \mathrm{N}$, which is higher than the value of $d_{33}=4.8 \mathrm{pC} / \mathrm{N}$ obtained for the composite sample (PZT/PVDF) without conductive phase, with $30 / 70$ vol. $\%$ poled in the same conditions. Samples with higher PZT/PAni contents could not be polarized because of breakdown, even in electric fields lower than $5.0 \mathrm{MV} / \mathrm{m}$.

For the PZT-PAni/PVDF composite, the poling electric field can be applied up to $10 \mathrm{MV} / \mathrm{m}$ even in samples with PZT-PAni content exceeding the percolation threshold. If the PZT-PAni content is below the critical concentration, e.g., 20 vol. $\%$, the electroactive property of the composite is low due to the small amount of ferroelectric phase in the composite film.

Fig. 5 shows the possible model of internal configuration for the composite samples obtained by the two different routes. In the PZT/PAni/PVDF composite (Fig. 5a), the electrical conduction path can appear without any contact or with little contact among the PZT particles, which makes the poling process difficult due to breakdown or because the electrical flux path passes through the sample. In the PZT-PAni/PVDF composite (Fig. 5b) (considering PZT totally coated with PAni), the electrical flux path is created over the particles. However, in this case, the direct current flux does not allow the particles to be polarized due to the shielding effect of the conductor phase on the particles, which prevents their contact with the applied electric field.
Fig. 5c shows what actually occurs. The PZT particles are only partially coated by the conductive phase, leaving islands of PAni on the PZT particles. The small distance between the island and the particle or between particles can generate capacitive and resistive effects, which are responsible for the poling process. Adjusting the impedance values [18] of PZT-PAni pellets with totally doped PAni (conductive state), as shown in Fig. 6, the equivalent electrical circuit should be represented by two circuits in series, each one with an associated resistor in parallel with a component with constant phase (EPC). The two circuits in series represent two relaxations, shown in the Cole graph [19, 20] (Fig. 6a) or in the impedance vs. frequency graph (Fig. 6b), which can be attributed to conduction or poling effects on or between the particles. The conduction path must necessarily cross the particles and inter-particle boundaries.

Is should be noted that the EPC component is practically a capacitor, since the value of $\mathrm{n}$ is close to 1 . However, no set for the ideal capacitor indicates that there are some resistive effects that cannot be adjusted. This suggests that there are alternative conduction paths and that the decrease in the electrical conductivity of the composite can enhance its polarization. However, if the electrical resistance is high, the resistance of the EPC will also be enhanced,

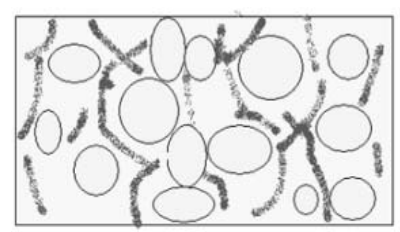

(a)

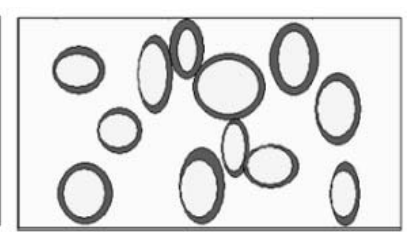

(b)

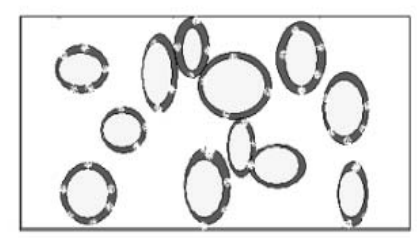

(c)

Fig. 5 Two-dimensional model for internal configuration over the percolation threshold: (a) PZT/PAni/PVDF; (b) PZT-PAni/PVDF with totally coated particles and (c) PZT-PAni/PVDF with partially coated particles. 


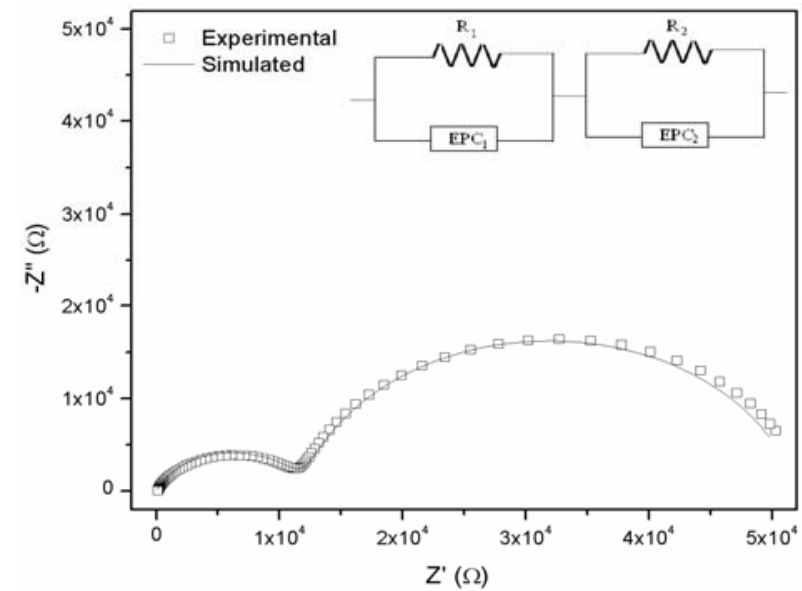

(a)

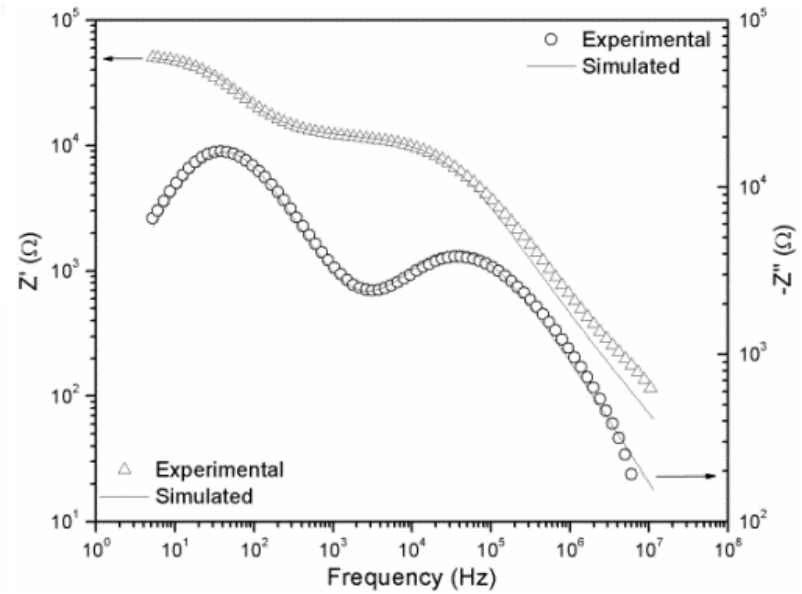

(b)

Fig. 6 Equivalent electrical circuit for PZT-PAni pellets. $R_{1}=1.20 \times 10^{4} \Omega ; Q=7.49 \times 10^{-9} C ; n_{1}=0.75 ; R_{2}=4.00 \times 10^{4} \Omega ; Q=$ $1.99 \times 10^{-7} \mathrm{C} ; \mathrm{n}_{2}=\mathbf{0 . 8 6}$. Electrode area $=0.35 \mathrm{~cm}^{2}$, thickness $=0.080 \mathrm{~cm}$. (a) Cole graph; (b) impedance $\mathrm{x}$ frequency graph.

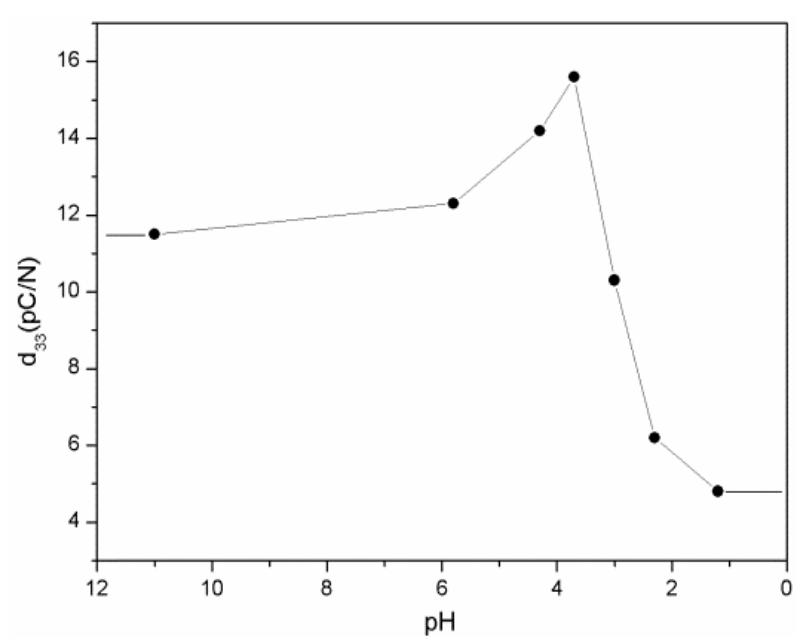

Fig. $7 \quad d_{33} \quad x \quad$ pH for PZT-PAni/PVDF $30 / 70$ vol.\% composite film.

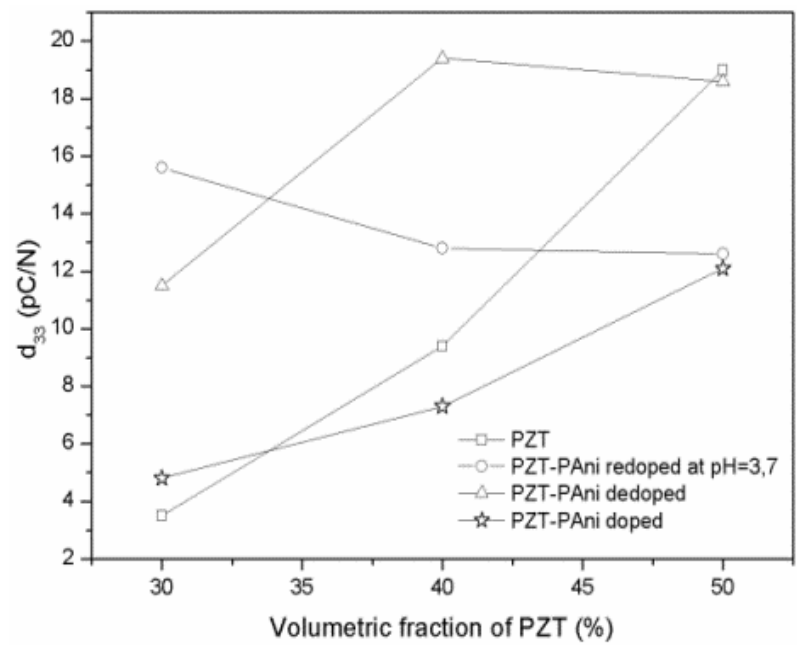

Fig. $8 d_{33} \times$ ceramic content: poling field: $5.0 \mathrm{MV} / \mathrm{m}$, poling time: $15 \mathrm{~min}$, and temperature of polarization: $90^{\circ} \mathrm{C}$. which hinders the poling process. Therefore, the ideal situation is an intermediate value at which the poling process can be optimized.

Fig. 7 shows the behavior of the longitudinal piezoelectric coefficient $d_{33}$ of PZT-PAni/PVDF at different levels of PAni doping. The sample was polarized with a $5.0 \mathrm{MV} / \mathrm{m}$ electric field for $15 \mathrm{~min}$ at $90^{\circ} \mathrm{C}$. Samples doped at $\mathrm{pH}=3.7$ showed the best results, reaching a $d_{33}$ value of $16.0 \mathrm{pC} / \mathrm{N}$. At $\mathrm{pH}$ values above and below 3.7, the $d_{33}$ values declined. The composite with dedoped PAni presented a $d_{33}$ coefficient of $11.0 \mathrm{pC} / \mathrm{N}$, while the fully doped composite showed a $d_{33}$ of only $4.0 \mathrm{pC} / \mathrm{N}$. With regard to the circuit (Fig. 6), it is clear that if the sample's electrical resistance decreases significantly, its preferential electrical flux path will be through the resistors. On the other hand, the electrical resistance of dedoped samples is high; albeit still lower than that of uncoated PZT particles.

Fig. 8 shows the $d_{33}$ behavior when the PZT-PAni ratio is changed. For comparison, the measurement of PZT/PVDF (without conducting phase) is also plotted. The composite containing PAni redoped at $\mathrm{pH}=3.7$ shows better results at 30 vol.\% of coated ceramic fillers. With higher PZT-PAni content, the piezoelectric coefficient decreases due to the increase in electrical conductivity. At 50 vol. $\%$, the $d_{33}$ value is 
the same as that of the fully doped PZT-PAni. The dedoped sample shows a better result at about 40 vol. $\%$ of PZT-PAni and, at $50 \mathrm{vol} . \%$, the piezoelectric coefficient is equal to $50 \mathrm{vol} \%$ of PZT/PVDF composite. It should be noted that a sample with this volume of ceramic content no longer shows pure 0-3 connectivity. The contact between coated particles increases the conduction channels, hindering the poling process. Also, it is more difficult to keep the polarized dipoles frozen after removing the polarization field.

Fig. 9 shows increasing electrical conductivity in composite films redoped at $\mathrm{pH}=3.7$, even at volume fractions of PZT-PAni above the percolation threshold, due to the increase in percolation channels, which occurs with increasing PZT-PAni content. The same explanation holds true for totally doped compositefilms.

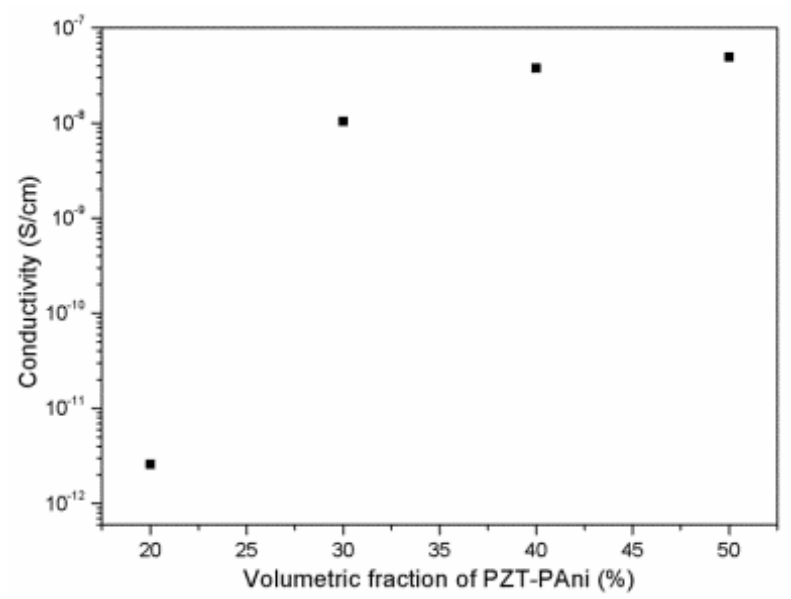

Fig. 9 Electrical conductivity in response to PZT-PAni content. PAni redoped at $\mathrm{pH}$ 3.7.

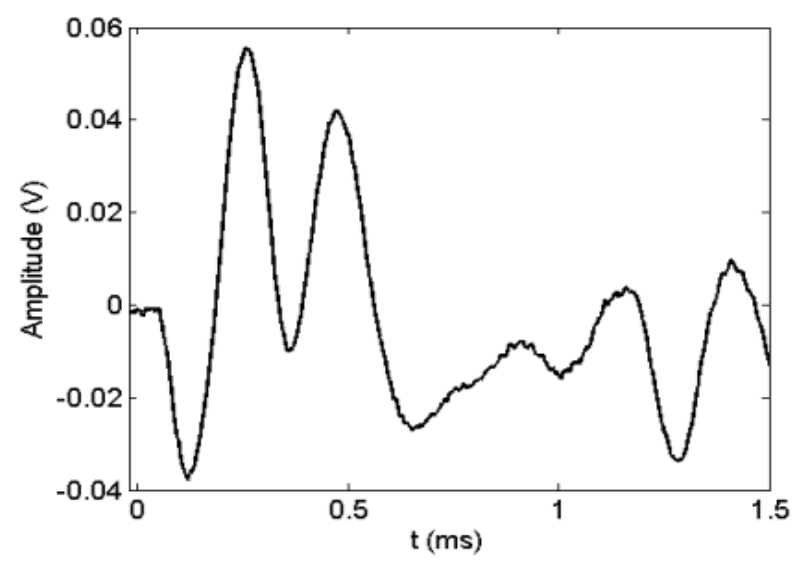

Fig. 10 Waveform in time domain. Response of the sensor to a pencil lead break.
The $d_{33}$ is enhanced with increasing PZT-PAni content because, essentially, the ferroelectric phase increases without a corresponding significant change in electrical conductivity (Fig. 3). Although the dedoped PZT-PAni/PVDF composite with 40 vol. $\%$ of ceramic particles showed a higher $d_{33}$ value, the sample with 30 vol. $\%$ of PZT-PAni redoped at $\mathrm{pH}=3.7$ seems to be the better choice, since the advantage of a lower ceramic content is that the flexibility of the composite film is preserved.

Using the best composite sample as sensor, it was surface mounted onto an aluminum plate for $\mathrm{AE}$ test. Fig. 10 shows acoustic waveform detected by an oscilloscope as a sensor response to a pencil lead break. It can be seen from the results obtained that PZT-PAni/PU composite poled with just $5.0 \mathrm{MV} / \mathrm{m}$ during $15 \mathrm{~min}$ has sensitivity enough to detect $\mathrm{AE}$ signal.

\section{Conclusions}

Composites with PZT particles partially coated with PAni and dispersed in PVDF matrix were prepared and their electrical conductivity was altered according to the degree of protonation of PAni. The poling efficiency was enhanced by controlling the conductivity of the composite samples. The percolation threshold for such composites is in the range of 20 to 30 vol\% of PZT-PAni inclusions. The electroactive property over the percolation threshold showed a better performance because the conductive phase coating was partial, allowing for polarization effects in the vicinity of the particles (pseudo-connection).

When PAni powder was dispersed as a third phase (separately from PZT particles) in the PVDF matrix, the percolation threshold varied from 0.5 vol. $\%$ to 1.0 vol.\% of PAni. Higher volumes of PAni prevented the sample from becoming polarized.

PZT-PAni/PVDF composite film redoped with hydrochloric acid at $\mathrm{pH}=3.7$ showed a high longitudinal piezoelectric coefficient, $d_{33}$, in samples containing $30 \mathrm{vol} . \%$ of ceramic due to the equilibrium 
between conduction and poling effects on the sample. The main advantage of using PZT particles coated with a conducting polymer is that flexible samples with low PZT content and good longitudinal piezoelectric coefficient can be obtained, resulting in a composite film with 0-3 connectivity. Furthermore, the lower poling electric field $(5 \mathrm{MV} / \mathrm{m})$ and the reduced poling time (15 $\mathrm{min}$ ) necessary for the polarization process of the composite film overcome the time consuming and the high electric voltage generally used with piezo composite.

Preliminary result indicates that this composite can be used as acoustic emission sensor. Characterization of the sensor is in development in our Lab.

\section{Acknowledgments}

The authors gratefully acknowledge the financial support of Fundação de Amparo à Pesquisa do Estado de São Paulo, FAPESP (São Paulo Research Foundation) through the INCTMN (National Institutefor Materials Science in Nanotechnology), Brazil. Thanks are duetoPro-Reitoria de Pesquisa, PROPe da UNESP.

\section{References}

[1] T. Furukawa, K. Ishida, E. Fukada, Piezoelectric properties in the composite systems of polymers and PZT ceramics, Journal of Applied Physics 50 (7) (1979) 4904-4912

[2] W.K. Sakamoto, S. Shibatta-Kagesawa, D.H.F. Kanda, S.H. Fernandes, E. Longo, G.O. Chierice, Piezoelectric effect in composite polyurethane-ferroelectric ceramics, Phys. Status Solidi A 5 (6) (2002) 257-260.

[3] J.R. White, B. De Poumeyrol, J.M. Hale, R. Stephenson, Piezoelectric paint: Ceramic-polymer composites for vibration sensors, Journal of Materials Science 39 (9) (2004) 3105-3114.

[4] W.K. Sakamoto, G.P. Estevam, A.A. De Carvalho, W. Pontes, M.H. De Paula, Ferroelectric ceramic/polymer composite for measuring X-ray intensity in the orthovoltage range, in: International Symposium on the Applications of Ferroelectrics, Nara, 2007, pp. 273-275.

[5] K.L. Ren, Y.M. Liu, X.C. Geng, H.F. Hofmann, Q.M. Zhang, Single crystal PMN-PT/Epoxy 1-3 composite for energy-harvesting application, IEEE Transactions on Ultrasonics, Ferroelectricsand Frequency Control. 53 (3)
(2006) 631-638.

[6] W.K. Sakamoto, G.C. Fuzari Jr., M.A. Zaghete, R.L.B. Freitas, Lead titanate-based nanocomposite: Fabrication, characterization and applications and energy conversion evaluation, Ferroelectrics Material Aspects, Intech, Rijeka-Croatia, 2011, pp. 251-276.

[7] X.F. Liu, C.X. Xiong, H.J. Sun, L.J. Dong, R. Li, Y. Liu, Piezoelectric and dielectric properties of PZT/PVC and graphite doped with PZT/PVC, Composites Materials Science and Engineering B Solid State Materials for Advanced Technology 127 (2-3) (2006) 261-266.

[8] G.C. Fuzari Jr, M.O. Orlandi, E. Longo, W.L.B. Melo, W.K. Sakamoto, Effect of controlled conductivity on thermal sensing property of 0-3 pyroelectric composite, Smart Materials and Structures 22 (2) (2013) 1-7.

[9] B. Ploss, M. Krause, Optimized pyroelectric properties of 0-3 composites of PZT particles in polyurethane doped with lithium perchlorate, IEEE Transactions on Ultrasonics Ferroelectrics and Frequency Control 54 (12) (2007) 2479-248.

[10] Y.M. Poon, F.G. Shin, A simple explicit formula for the effective dielectric constant of binary 0-3 composites, Journal of Materials Science 39 (4) (2004) 1277-128.

[11] B. Ploss, Y.W. Wong, F.G. Shin, Pyroelectric ceramic/polymer composite with electrically conducting matrix material, Ferroelectrics 325 (2005) 165-169.

[12] C.K. Wong, F.G. Shin, Effect of electrical conductivity on poling and the dielectric, pyroelectric and piezoelectric properties of ferroelectric 0-3 composites, Journal of Materials Science 41 (1) (2006) 229-249.

[13] M.P. Wenger, P. Blanas, R.J. Shuford, D.K. Das-Gupta, Characterization and evaluation of piezoelectric composite bimorph for in situ acoustic emission sensors, Polym. Eng. Sci. 39 (3) (1999) 508-518.

[14] R.E. Newnham, D.P. Skinner, L.E. Cross, Connectivity and piezoelectric-pyroelectric composites, Materials Research Bulletin 13 (5) (1978) 525-523.

[15] A. Bunde, W. Dieterich, Percolation in Composites, Journal of Electroceramics 5 (2) (2000) 81-92.

[16] G. Macdiarmid, J.C. Chiang, A.F. Richter, Polyaniline: a new concept in conducting polymers, Synthetic Metals 18 (1-3) (1987) 28- 290.

[17] A. Balberg, D. Azulay, D. Toker, O. Millo, Percolation and tunneling in composite materials, International Journal of Modern Physics B18 (15) (2004) 2091-2121.

[18] A. Boukamp, A nonlinear least squares fit procedure for analysis of immittance data of electrochemical systems, Solid State Ionics 20 (1) (1986) 31-44.

[19] R.H. Cole, Relaxation processes in dielectrics, Journal of Cellular and Comparative Physiology 66 (3) (1965) 13-25.

[20] K. Jonscher, The universal dielectric response, Nature 267 (5613) (1977) 673-679. 